\title{
On the existence problem of solutions to a class of fuzzy mixed exponential vector variational inequalities
}

\author{
Shih-Sen Chang ${ }^{\mathrm{a}}$, Salahuddin ${ }^{\mathrm{b}}$, Ching-Feng Wen ${ }^{\mathrm{c}, \mathrm{d}, *}$, Xiong Rui Wang $^{\mathrm{e}}$ \\ ${ }^{a}$ Center for General Education, China Medical University, Taichung 40402, Taiwan. \\ ${ }^{b}$ Department of Mathematics, Jazan University, Jazan, Kingdom of Saudi Arabia. \\ ${ }^{c}$ Center for Fundamental Science; and Research Center for Nonlinear Analysis and Optimization, Kaohsiung Medical University, \\ Kaohsiung 80708, Taiwan.
}

${ }^{d}$ Department of Medical Research, Kaohsiung Medical University Hospital, Kaohsiung 80708, Taiwan.

${ }^{e}$ Department of Mathematics, Yibin University, Yibin, Sichuan 644007, China.

Communicated by P. Kumam

\begin{abstract}
In this research article, we deal with a new kind of mixed exponential fuzzy vector variational inequalities in ordered Euclidean spaces. By using KKM-technique and Nadler's fixed point theorem, we prove some existence theorems of solutions to mixed exponential vector variational inequality problems in fuzzy environment.
\end{abstract}

Keywords: Mixed exponential vector variational inequality problems, fuzzy mappings, fuzzy upper and lower semicontinuous mappings, $\alpha_{g}$-relaxed exponentially $(\gamma, \eta)$-monotone mapping, KKM-mappings, Nadler's fixed points theorem, ordered Euclidean spaces.

2010 MSC: 47H09, 47J20.

(C)2018 All rights reserved.

\section{Introduction}

The concept of fuzziness was first discovered and introduced in the seminal article written by Zadeh in 1965 [32]. The theory of fuzzy topological spaces was introduced and initiated by Chang [8] and since then various notions in classical topology have been extended to fuzzy topological spaces. In 1989, Chang and Zhu [11] introduced the concepts of variational inequalities with fuzzy mappings in abstract spaces and investigated the existence problems of solutions for some class of variational inequalities for fuzzy mappings. Recently several classes of variational inequalities, inclusions, and complementarity problems for fuzzy mappings were studied by Ahmad and Salahuddin [1, 3], Chang and Salahuddin [9], Cho et al. [14], Ding et al. [16], Ding and Salahuddin [15], Lee and Salahuddin [28], Verma and Salahuddin [29], Verma et al. [27], and Wang and Chang et al. [10].

\footnotetext{
*Corresponding author

Email address: cf wen@kmu.edu.tw (Ching-Feng Wen)

doi: $10.22436 /$ jnsa.011.07.04
} 
In 1980, Giannessi [19] first introduced the concept of vector variational inequality in a finite dimensional Euclidean spaces. Since then many kinds of vector variational inequalities have been introduced and studied in abstract spaces. In particular, Chen and Cheng [13], Ahmad and Salahuddin [2], Anastassiou and Salahuddin [4], Anh and Khanh [5], Khanh and Quan [22], Lee and Salahuddin [23, 24], and Siddiqi et al. [30] have intensively studied some kinds of vector variational inequalities in abstract spaces and obtained some existence theorems of solutions for their variational inequalities.

In the study of vector variational inequality problems, the assumption of generalized monotonicity of the operators plays a very important role. Wu and Huang [31] introduced the concepts of relaxed $\eta$ - $\alpha$-pseudomonotonicity mappings and use this concept to study the vector variational like inequalities in Banach spaces. Ceng and Yao [7] studied the generalized variational like inequality problems with generalized $\alpha$-monotone multifunction. Very recently, Verma et al. [21] studied the exponential type vector variational like inequality problems with exponential invexities.

Motivated by the researches going on in this directions $([6,12,15,18,20,25,26])$, the purpose of this paper is to introduce and study a new class of mixed exponential vector variational inequality problems and define a new class of $\alpha_{\mathrm{g}}$-relaxed exponential $(\gamma, \eta)$-monotone mappings in fuzzy environment. We prove the existence of solutions to mixed exponential vector variational inequality problems with fuzzy mappings by using the KKM-mapping and Nadler's fixed point theorem.

\section{2. preliminaries}

Let $E$ be a nonempty subset of a vector space $X$ and $D$ be a nonempty set. A mapping $F$ from $D$ into $\mathfrak{F}(E)$ (the collection of all fuzzy sets of $E$ ) is called a fuzzy mapping. If $F: D \rightarrow \mathfrak{F}(E)$ is a fuzzy mapping, then $F(x), x \in D$ (denoted by $F_{x}$, in the sequel) is a fuzzy set in $\mathfrak{F}(E)$ and $F_{x}(y), y \in E$ is the grade of membership of $y$ in $F_{x}$.

Let $A \in \mathfrak{F}(E)$ and $\beta \in[0,1]$, then the set

$$
(A)_{\beta}=\{x \in E: A(x) \geqslant \beta\}
$$

is called a $\beta$-cut set of $A$.

In the sequel, we assume that $X$ and $Y$ are real Banach spaces and $K$ is a nonempty subset of $X$.

A nonempty subset $C$ of $Y$ is called convex cone if $\lambda C \subset C$ for any $\lambda \geqslant 0$ and $C+C \subset C$. $C$ is called pointed cone if $C$ is a convex cone and $C \cap\{-C\}=\{0\}$, where 0 denotes the zero vector. Also a cone $C$ is called proper if it is properly contained in $\mathrm{Y}$. Let $\mathrm{C}: \mathrm{K} \rightarrow 2^{\mathrm{Y}}$ be a multifunction such that for each $x \in K, C(x)$ is closed convex moving cone with apex at origin and $\operatorname{intC}(x) \neq \emptyset$, where intC $(x)$ denotes the interior of $\mathrm{C}(\mathrm{x})$. The partial ordering $\leqslant \mathrm{C}(\mathrm{x})$ on $\mathrm{Y}$ induced by $\mathrm{C}(\mathrm{x})$ is defined as

1. $\mathrm{y} \leqslant \mathrm{C}(\mathrm{x}) z \Leftrightarrow z-\mathrm{y} \in \mathrm{C}(\mathrm{x})$;

2. $\mathrm{y} \notin \mathrm{C}(\mathrm{x}) z \Leftrightarrow z-\mathrm{y} \notin \mathrm{C}(\mathrm{x})$;

3. $y \leqslant \operatorname{intC}(x) z \Leftrightarrow z-y \in \operatorname{intC}(x)$;

4. $y \operatorname{kintC}_{(x)} z \Leftrightarrow z-y \notin \operatorname{intC}(x)$ for $x, y, z \in K$;

5. $y \nless \mathrm{C}_{(x)} z \Leftrightarrow y+w \notin \mathrm{C}(\mathrm{x}) z+w$ for all $x, y, z, w \in K$;

6. $y \not c(x) z \Leftrightarrow \lambda y \not c(x) \lambda z$ for any $\lambda \geqslant 0$.

Lemma 2.1 ([7]). Let $(\mathrm{Y}, \mathrm{C})$ be an ordered space induced by the pointed closed convex cone $\mathrm{C} \subset \mathrm{Y}$ with int $\mathrm{C} \neq \emptyset$. Then for any $x, y, z \in Y$, the following relations hold:

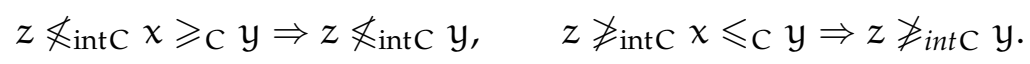

Denote by $L(X, Y)$ the space of all continuous linear mappings from $X$ to $Y$ and by $\langle u, x\rangle$ the evaluation of $u \in L(X, Y)$ at $x \in X$. Let $T: X \rightarrow 2^{Y}$ be a multifunction. The graph $\mathfrak{G}(T)$ of $T$ is the set $\{(x, y) \in X \times Y:$ $x \in X, y \in T(x)\}$. The inverse $T^{-1}$ of $T$ is a multifunction from $R(T)$ (the range of $T$ ) to $X$. 
In the following we assume that $\mathrm{K}$ is a nonempty closed convex subset of $\mathrm{X}$, and $(\mathrm{Y}, \mathrm{C})$ is an ordered Euclidean space induced by a closed convex pointed cone valued mapping $C: K \rightarrow 2^{Y}$, whose apex at origin and $\operatorname{int} C(x) \neq \emptyset$. Let $\gamma \in R$ be a nonzero real number, $\eta: K \times K \rightarrow X, g: K \rightarrow K$ and $f: K \times K \rightarrow Y$ be the mappings, and $N: \mathfrak{F}(L(X, Y)) \rightarrow \mathfrak{F}(L(X, Y))$ be a mapping. Let $T: K \rightarrow \mathfrak{F}(L(X, Y))$ be a fuzzy mapping on $2^{\mathrm{L}(X, Y)}$ and $a: K \rightarrow[0,1]$ be a function. Then the mixed exponential vector variational inequality problems with fuzzy mapping is to find $x \in K, u \in \tilde{T}(x)=(T x)_{a(x)}$ such that

$$
\left\langle\mathrm{Nu}, \frac{1}{\gamma}\left(e^{\gamma \eta(y, g(x))}-1\right)\right\rangle+\mathrm{f}(\mathrm{g}(\mathrm{x}), \mathrm{y}) \mathbb{i n t C}_{\mathrm{int}} 0, \forall \mathrm{y} \in \mathrm{K}
$$

Definition 2.2. A mapping $f: X \rightarrow Y$ is said to be $C(x)$-convex on $X$, if

$$
f(t x+(1-t) y) \leqslant c(x) t f(x)+(1-t) f(y), \forall x, y \in X, t \in[0,1]
$$

i.e.,

$$
t f(x)+(1-t) f(y)-f(t x+(1-t) y) \in C(x), \forall x, y \in X, t \in[0,1] .
$$

Remark 2.3. When $\mathrm{C}(\mathrm{x})=\mathrm{C}$, then $\mathrm{f}: \mathrm{X} \rightarrow \mathrm{Y}$ is said to be $\mathrm{C}$-convex on $\mathrm{X}$, if

$$
f(t x+(1-t) y) \leqslant c t f(x)+(1-t) f(y), \forall x, y \in X, t \in[0,1] .
$$

Definition 2.4. A mapping $f: K \rightarrow Y$ is said to be the completely continuous if for any sequence $\left\{x_{n}\right\} \in K$, $x_{\mathrm{n}} \rightarrow x_{0} \in K$ weakly, then $\mathrm{f}\left(\mathrm{x}_{\mathrm{n}}\right) \rightarrow \mathrm{f}\left(\mathrm{x}_{0}\right)$.

Definition 2.5. Let $f: K \rightarrow 2^{X}$ be a set valued mapping. Then $f$ is said to be a KKM-mapping if for any $\left\{y_{1}, y_{2}, \ldots, y_{n}\right\}$ of $K$, we have

$$
\operatorname{co}\left\{y_{1}, y_{2}, \ldots, y_{n}\right\} \subset \bigcup_{i=1}^{n} f\left(y_{i}\right),
$$

where $\operatorname{co}\left\{y_{1}, y_{2}, \ldots, y_{n}\right\}$ denotes the convex hull of $y_{1}, y_{2}, \ldots, y_{n}$.

Lemma 2.6 ([17]). Let $M$ be a nonempty subset of a Hausdorff topological vector space $\mathrm{X}$ and let $\mathrm{f}: \mathrm{M} \rightarrow 2^{\mathrm{X}}$ be a $K K M$-mapping. If for each $\mathrm{y} \in \mathrm{M}, \mathrm{f}(\mathrm{y})$ is a closed set in $\mathrm{X}$ and for some $\mathrm{y} \in \mathrm{M}, \mathrm{f}(\mathrm{y})$ is compact, then

$$
\bigcap_{y \in M} f(y) \neq \emptyset .
$$

Lemma 2.7 ([26]). Let $\mathrm{E}$ be a normed vector space and $\mathrm{H}$ be a Hausdorff metric on the collection $\mathrm{CB}(\mathrm{E})$ of all closed and bounded subsets of $\mathrm{E}$ defined by

$$
H(A, B)=\max \left\{\sup _{x \in A} \inf _{y \in B}\|x-y\|, \sup _{y \in B} \inf _{x \in A}\|x-y\|\right\}, A, B \in C B(E) .
$$

(1) If $\mathrm{A}, \mathrm{B} \in \mathrm{CB}(\mathrm{E})$, then for each $\epsilon>0$ and each $\mathrm{x} \in \mathrm{A}$, there exists $\mathrm{y} \in \mathrm{B}$ such that

$$
\|x-y\| \leqslant(1+\epsilon) H(A, B) .
$$

(2) If $\mathrm{A}$ and $\mathrm{B}$ are compact subset in $\mathrm{E}$, then for each $\mathrm{x} \in \mathrm{A}$, there exists $\mathrm{y} \in \mathrm{B}$ such that

$$
\|x-y\| \leqslant H(A, B) .
$$

Definition 2.8. Let $K$ be a nonempty closed convex subset of $X, \eta: X \times X \rightarrow X$ be a mapping, and $N: \mathfrak{F}(L(X, Y)) \rightarrow \mathfrak{F}(L(X, Y))$ be a single valued mapping, where $L(X, Y)$ is the space of all continuous linear mappings from $X$ to $Y$. Suppose that $T: K \rightarrow \mathfrak{F}(L(X, Y))$ is a fuzzy mapping with $(T x)_{a}(x) \neq \emptyset$ for all $x \in K$, where $a: X \rightarrow[0,1]$ and $\tilde{T}: K \rightarrow 2^{L(X, Y)}$ is a nonempty compact set valued mapping defined by $\tilde{T}(x)=(T x)_{a(x)}$, then 
(i) $\mathrm{N}$ is said to be $\eta$-hemicontinuous if

$$
\lim _{t \rightarrow 0^{+}}\langle N(x+t(y-x)), \eta(y, x)\rangle=\langle N x, \eta(y, x)\rangle \text { for each } x, y \in K .
$$

(ii) $T$ is said to be H-hemicontinuous, if for any given $x, y \in K$, the mapping $t \rightarrow H(T(x+t(y-x))$, Tx) is continuous at $0^{+}$, where $\mathrm{H}$ is the Hausdorff matric defined on $\mathrm{CB}(\mathrm{L}(\mathrm{X}, \mathrm{Y})$ ).

Definition 2.9. A mapping $f: X \rightarrow X$ is said to be affine if for any $x_{i} \in K$ and $\lambda_{i} \geqslant 0,(1 \leqslant i \leqslant n)$ with $\sum_{i=1}^{n} \lambda_{i}=1$, we have

$$
f\left(\sum_{i=1}^{n} \lambda_{i} x_{i}\right)=\sum_{i=1}^{n} \lambda_{i} f\left(x_{i}\right) .
$$

Definition 2.10. Let $X$ be an Euclidean space. A function $f: X \rightarrow R$ is a lower semicontinuous at $x_{0} \in X$ if

$$
f\left(x_{0}\right) \leqslant \lim \inf _{n} f\left(x_{n}\right)
$$

for any sequence $\left\{x_{n}\right\} \subset X$ such that $\left\{x_{n}\right\}$ converges to $x_{0}$.

Definition 2.11. Let $X$ be an Euclidean space. A function $f: X \rightarrow R$ is said to be weakly upper semicontinuous at $x_{0} \in X$, if

$$
f\left(x_{0}\right) \geqslant \lim \sup _{n} f\left(x_{n}\right)
$$

for any sequence $\left\{x_{n}\right\} \in X$ such that $\left\{x_{n}\right\}$ converges to $x_{0}$ weakly.

Lemma 2.12 ([6]). Let $\mathrm{S}$ be a nonempty, compact, and convex subset of a finite dimensional space and $\mathrm{T}: \mathrm{S} \rightarrow \mathrm{S}$ be a continuous mapping. Then there exists $x \in S$ such that $\mathrm{T}(\mathrm{x})=\mathrm{x}$.

Definition 2.13. Let $X, Y$ be topological spaces and $T: X \rightarrow \mathfrak{F}(Y)$ be a fuzzy mapping. $T$ is said to have fuzzy set valued if $T_{x}(y)$ is upper semi continuous on $X \times Y$ as a ordinary real function.

Lemma 2.14 ([6]). Let A be a closed subset of a topological space $X$, then characteristic function $\chi_{\mathrm{A}}$ of $\mathrm{A}$ is an upper semi continuous real valued function.

Lemma 2.15 ([6, 10]). Let $\mathrm{K}$ be a nonempty closed convex subset of a real Hausdorff topological vector space $\mathrm{X}$, $\mathrm{E}$ be a nonempty closed convex subset of a real Hausdorff space, and $\mathrm{a}: \mathrm{X} \rightarrow[0,1]$ be a lower semi continuous function. Let $\mathrm{T}: \mathrm{K} \rightarrow \mathfrak{F}(\mathrm{E})$ be a fuzzy mapping with $(\mathrm{T} x)_{\mathrm{a}(\mathrm{x})} \neq \emptyset$ for all $\mathrm{x} \in \mathrm{X}$ and $\tilde{\mathrm{T}}: \mathrm{K} \rightarrow 2^{\mathrm{E}}$ be a multifunction defined by $\tilde{\mathrm{T}}(\mathrm{x})=(\mathrm{T} \mathrm{x})_{\mathrm{a}(\mathrm{x})}$. If $\mathrm{T}$ is a closed set valued mapping, then $\tilde{\mathrm{T}}$ is a closed multifunction.

Definition 2.16. A fuzzy mapping $T: K \rightarrow \mathfrak{F}(L(X, Y))$ is said to be $\alpha_{g}$-relaxed exponentially $(\gamma, \eta)$ monotone if for every pair of points $x, y \in K$, we have

$$
\left\langle\mathrm{T} x-\mathrm{T} y, \frac{1}{\gamma}\left(e^{\gamma \eta(x, g(y))}-1\right)\right\rangle \geqslant_{\mathrm{C}(x)} \alpha_{\mathrm{g}}(x-y),
$$

where $\alpha_{g}: X \rightarrow Y$ with $\alpha_{g}(t x)=t^{q} \alpha_{g}(x)$ for all $t>0$ and $x \in X$, where $q>1$ is a real number.

Definition 2.17. Let $N: \mathfrak{F}(L(X, Y)) \rightarrow \mathfrak{F}(L(X, Y))$ be a single valued mapping and $a: X \rightarrow[0,1]$ be a mapping. A fuzzy mapping $T: K \rightarrow \mathfrak{F}(L(X, Y))$ with compact valued is said to be $\alpha_{g}$-relaxed exponentially $(\gamma, \eta)$-monotone with respect to $N$ and $g$, if for each pair of points $x, y \in K$, we have

$$
\left\langle\mathrm{Nu}-\mathrm{N} v, \frac{1}{\gamma}\left(\mathrm{e}^{\gamma \eta(x, g(y))}-1\right)\right\rangle \geqslant_{\mathrm{C}(x)} \alpha_{\mathrm{g}}(x-y), \forall u \in(\mathrm{Tx})_{\mathrm{a}(\mathrm{x})}, v \in(\mathrm{Ty})_{\mathrm{a}(\mathrm{y})},
$$

where $\alpha_{g}: X \rightarrow Y$ with $\alpha_{g}(t x)=t^{q} \alpha_{g}(x)$ for all $t>0$ and $x \in X$, where $q>1$ is a real number. 


\section{Main results}

Theorem 3.1. Let $\mathrm{K}$ be a nonempty closed convex and bounded subset of a real ordered Euclidean space $\mathrm{X}$. Let $\mathrm{C}: \mathrm{K} \rightarrow 2^{\mathrm{Y}}$ be a closed convex bounded cone valued mapping with int $\mathrm{C} \neq \emptyset$ and $\mathrm{Y} \backslash(-\operatorname{int} \mathrm{C}(\mathrm{x}))$ being an upper semi continuous mapping. Let $\mathrm{g}: \mathrm{K} \rightarrow \mathrm{K}$ be a closed convex and continuous single valued mapping and $\eta: \mathrm{K} \times \mathrm{K} \rightarrow \mathrm{X}$ be an affine in the first argument with $\eta(x, g(x))=0$ for all $\mathrm{x} \in \mathrm{K}$. Let $\mathrm{f}: \mathrm{K} \times \mathrm{K} \rightarrow \mathrm{Y}$ be a $\mathrm{C}(\mathrm{x})$-convex in the second argument satisfying $\mathrm{f}(\mathrm{g}(\mathrm{x}), \mathrm{x})=0$ for all $\mathrm{x} \in \mathrm{K}$. Let $\mathrm{N}: \mathfrak{F}(\mathrm{L}(\mathrm{X}, \mathrm{Y})) \rightarrow \mathfrak{F}(\mathrm{L}(\mathrm{X}, \mathrm{Y}))$ be a continuous mapping and let $\tilde{\mathrm{T}}: \mathrm{K} \rightarrow 2^{\mathrm{L}(\mathrm{X}, \mathrm{Y})}$ be a nonempty upper semi continuous compact valued mapping induced by fuzzy mapping $\mathrm{T}: \mathrm{K} \rightarrow \mathfrak{F}(\mathrm{L}(\mathrm{X}, \mathrm{Y}))$, i.e. $\tilde{\mathrm{T}}(\mathrm{x})=(\mathrm{T} x)_{\mathrm{a}(\mathrm{x})}$ with $\mathrm{a}: \mathrm{X} \rightarrow[0,1]$. If $\tilde{\mathrm{T}}$ is $\mathrm{H}$-hemicontinuous and $\alpha_{\mathrm{g}}$-relaxed exponentially $(\gamma, \eta)$-monotone with respect to $\mathrm{N}$ and $\mathrm{g}$, then the following two statements $(\mathrm{a})$ and $(\mathrm{b})$ are equivalent:

(a) there exists $\bar{x} \in \mathrm{K}$ and $\overline{\mathrm{u}} \in \tilde{\mathrm{T}}(\overline{\mathrm{x}})=(\mathrm{T} x)_{\mathrm{a}(\mathrm{x})}$ such that

$$
\left\langle\mathrm{N} \bar{u}, \frac{1}{\gamma}\left(e^{\gamma \eta(y, g(\bar{x}))}-1\right)\right\rangle+f(g(\bar{x}), y) z_{\operatorname{int} C}(x) 0, \forall y \in K ;
$$

(b) there exists $\bar{\chi} \in \mathrm{K}$ such that

$$
\left\langle\mathrm{N} v, \frac{1}{\gamma}\left(e^{\gamma \eta(y, g(\bar{x}))}-1\right)\right\rangle+f(g(\bar{x}), y) \mathbb{i n t C}(x)_{g} \alpha_{g}(y-\bar{x}) \forall y \in K, v \in \tilde{T}(y)=(T y)_{a(y)} .
$$

Proof. Let the statement (a) is true, i.e., there exist $\bar{x} \in K$ and $\bar{u} \in \tilde{T}(\bar{x})=(T \bar{x})_{a(\bar{x})}$ such that

$$
\left\langle\mathrm{N} \bar{u}, \frac{1}{\gamma}\left(e^{\gamma \eta(y, g(\bar{x}))}-1\right)\right\rangle+f(g(\bar{x}), y) \operatorname{intC}_{(\bar{x})} 0, \quad \forall y \in K .
$$

Since $\tilde{T}$ is a $\alpha_{g}$-relaxed exponentially $(\gamma, \eta)$-monotone with respect to $N$ and $g$, we have

$$
\begin{aligned}
& \left\langle N v-N \bar{u}, \frac{1}{\gamma}\left(e^{\gamma \eta(y, g(\bar{x}))}-1\right)\right\rangle+f(g(\bar{x}), y) \geqslant c(\bar{x}) \alpha_{g}(y-\bar{x})+f(g(\bar{x}), y), \quad \forall y \in K, v \in \tilde{T}(y)=(T y)_{a(y)}, \\
& \Rightarrow\left\langle\mathrm{N} v, \frac{1}{\gamma}\left(e^{\gamma \eta(y, g(\bar{x}))}-1\right)\right\rangle+\mathrm{f}(\mathrm{g}(\bar{x}), y) \geqslant_{C}(\bar{x})\left\langle N \bar{u}, \frac{1}{\gamma}\left(e^{\gamma \eta(y, g(\bar{x}))}-1\right)\right\rangle \\
& +\alpha_{g}(y-\bar{x})+f(g(\bar{x}), y), \quad \forall y \in K, v \in \tilde{T}(y)=(T y)_{a(y)} \\
& \Rightarrow\left\langle\mathrm{N} v, \frac{1}{\gamma}\left(e^{\gamma \eta(y, g(\bar{x}))}-1\right)\right\rangle+\mathrm{f}(\mathrm{g}(\bar{x}), y)-\alpha_{g}(y-\bar{x}) \geqslant \mathrm{C}(\bar{x})\left\langle N \bar{u}, \frac{1}{\gamma}\left(e^{\gamma \eta(y, g(\bar{x}))}-1\right)\right\rangle \\
& +f(g(\bar{x}), y), \forall y \in K, v \in \tilde{T}(y)=(T y)_{a}(y) .
\end{aligned}
$$

From (3.1), (3.2), and Lemma 2.1, we get

$$
\left\langle\mathrm{N} v, \frac{1}{\gamma}\left(e^{\gamma \eta(y, g(\bar{x}))}-1\right)\right\rangle+f(g(\bar{x}), y) \gtrless_{i n t} \mathrm{C}(\bar{x}) \alpha_{g}(y-\bar{x}), \quad \forall y \in K, v \in \tilde{T}(y)=(T y)_{a}(y) .
$$

Conversely, assume that the statement (b) is true, i.e., there exists $\bar{x} \in \mathrm{K}$ such that

$$
\left\langle\mathrm{N} v, \frac{1}{\gamma}\left(e^{\gamma \eta(y, g(\bar{x}))}-1\right)\right\rangle+f(g(\bar{x}), y) \gtrless_{\operatorname{int} C(\bar{x})} \alpha_{g}(y-\bar{x}), \quad \forall y \in K, v \in \tilde{T}(y)=(T y)_{a}(y) .
$$

Let $y \in K$ be any point and $y_{t}=t y+(1-t) \bar{x}, t \in[0,1]$. Since $K$ is convex, $y_{t} \in K$. Let $v_{t} \in \tilde{T}\left(y_{t}\right)_{a}\left(y_{t}\right)$, from (3.3) we have

$$
\left\langle N v_{t}, \frac{1}{\gamma}\left(e^{\gamma \eta\left(y_{t}, g(\bar{x})\right)}-1\right)\right\rangle+f\left(g(\bar{x}), y_{t}\right) \operatorname{kint}_{(\bar{x})} \alpha_{g}\left(y_{t}-\bar{x}\right)=t^{q} \alpha_{g}(y-\bar{x}) .
$$

However, since

$$
\left\langle N v_{t}, \frac{1}{\gamma}\left(e^{\gamma \eta\left(y_{t}, g(\bar{x})\right)}-1\right)\right\rangle+f\left(g(\bar{x}), y_{t}\right)
$$




$$
\begin{aligned}
& =\left\langle\mathrm{N} v_{\mathrm{t}}, \frac{1}{\gamma}\left(e^{\gamma \eta(\mathrm{ty}+(1-\mathrm{t}) \bar{x}, \mathrm{~g}(\bar{x}))}-1\right)\right\rangle+\mathrm{f}(\mathrm{g}(\overline{\mathrm{x}}), \mathrm{ty}+(1-\mathrm{t}) \overline{\mathrm{x}}) \\
& =\left\langle\mathrm{N} v_{\mathrm{t}}, \frac{1}{\gamma}\left(e^{\gamma \operatorname{t\eta }(y, g(\bar{x}))+(1-\mathrm{t}) \gamma \eta(\bar{x}, g(\bar{x}))}-1\right)\right\rangle+\operatorname{tf}(g(\bar{x}), y)+(1-t) f(g(\bar{x}), \bar{x}) \\
& \leqslant C(\bar{x})\left\langle N v_{t}, \frac{1}{\gamma}\left(t\left(e^{\gamma \eta(y, g(\bar{x}))}-1\right)+(1-t)\left(e^{\gamma \eta(\bar{x}, g(\bar{x}))}-1\right)\right)\right\rangle+\operatorname{tf}(g(\bar{x}), y) \\
& =\mathrm{t}\left\{\left\langle\mathrm{N} v_{\mathrm{t}}, \frac{1}{\gamma}\left(e^{\gamma \eta(y, g(\bar{x}))}-1\right)\right\rangle+\mathrm{f}(\mathrm{g}(\bar{x}), y)\right\},
\end{aligned}
$$

it follows from (3.4), (3.5), and Lemma 2.1 that

$$
\left\langle N v_{t}, \frac{1}{\gamma}\left(e^{\gamma \eta(y, g(\bar{x}))}-1\right)\right\rangle+f(g(\bar{x}), y) \mathbb{i n t C}(\bar{x}) t^{q-1} \alpha_{g}(y-\bar{x})
$$

Since $\tilde{T}\left(y_{t}\right)=\left(T y_{t}\right)_{a\left(y_{t}\right)}$ and $\tilde{T}(\bar{x})=(T \bar{x})_{a}(\bar{x})$ are compact, from Lemma 2.7, for each fixed $v_{t} \in \tilde{T}\left(y_{t}\right)=$ $\left(T y_{t}\right)_{a\left(y_{t}\right)}$, there exists $u_{t} \in \tilde{T}(\bar{x})=(T \bar{x})_{a}(\bar{x})$ such that

$$
\left\|v_{t}-u_{t}\right\| \leqslant H\left(\tilde{T}\left(y_{t}\right), \tilde{T}(\bar{x})\right) .
$$

Since $\tilde{T}(\bar{x})$ is compact, without loss of generality, we may assume that

$$
\mathrm{u}_{\mathrm{t}} \rightarrow \overline{\mathrm{u}} \in \tilde{\mathrm{T}}(\overline{\mathrm{x}}) \text { as } \mathrm{t} \rightarrow 0^{+} .
$$

Also $\tilde{\mathrm{T}}$ is H-hemicontinuous, thus it follows that

$$
\mathrm{H}\left(\tilde{\mathrm{T}}\left(\mathrm{y}_{\mathrm{t}}\right), \tilde{\mathrm{T}}(\overline{\mathrm{x}})\right) \rightarrow 0 \text { as } \mathrm{t} \rightarrow 0^{+} .
$$

Now from (3.6) we have

$$
\left\|v_{t}-\bar{u}\right\| \leqslant\left\|v_{t}-u_{t}\right\|+\left\|u_{t}-\bar{u}\right\| \leqslant H\left(\tilde{T}\left(y_{t}\right), \tilde{T}\left(x_{t}\right)\right)+\left\|u_{t}-\bar{u}\right\| \rightarrow 0 \text { as } t \rightarrow 0^{+} .
$$

Since $\mathrm{N}$ is continuous, letting $t \rightarrow 0^{+}$, we have

$$
\begin{aligned}
\|\left\langle N v_{t}\right. & \left.\frac{1}{\gamma}\left(e^{\gamma \eta(y, g(\bar{x}))}-1\right)\right\rangle-t^{q-1} \alpha_{g}(y-\bar{x})-\left\langle N \bar{u}, \frac{1}{\gamma}\left(e^{\gamma \eta(y, g(\bar{x}))}-1\right)\right\rangle \| \\
& \leqslant\left\|\left\langle N v_{t}-N \bar{u}, \frac{1}{\gamma}\left(e^{\gamma \eta(y, g(\bar{x}))}-1\right)\right\rangle\right\|+\left\|t^{q-1} \alpha_{g}(y-\bar{x})\right\| \\
& \leqslant \frac{1}{\gamma}\left\|N v_{t}-N \bar{u}\right\|\left\|e^{\gamma \eta(y, g(\bar{x}))}-1\right\|+t^{q-1}\left\|\alpha_{g}(y-\bar{x})\right\| \rightarrow 0 \text { as } t \rightarrow 0^{+} .
\end{aligned}
$$

From (3.4), we have

$$
\left\langle\mathrm{N} v_{\mathrm{t}}, \frac{1}{\gamma}\left(e^{\gamma \eta(y, g(\bar{x}))}-1\right)\right\rangle+\mathrm{f}(\mathrm{g}(\bar{x}), y)-\mathrm{t}^{\mathrm{q}-1} \alpha_{\mathrm{g}}(\mathrm{y}-\overline{\mathrm{x}}) \in \mathrm{Y} \backslash(-\operatorname{intC}(\bar{x})) .
$$

Since $Y \backslash(-\operatorname{int} C(\bar{x}))$ is closed, from (3.7) we have

$$
\begin{aligned}
& \left\langle\mathrm{N} \bar{u}, \frac{1}{\gamma}\left(e^{\gamma \eta(y, g(\bar{x}))}-1\right)\right\rangle+f(g(\bar{x}), y) \in Y \backslash(-\operatorname{intC}(\bar{x})) \\
& \Longrightarrow\left\langle N \bar{u}, \frac{1}{\gamma}\left(e^{\gamma \eta(y, g(\bar{x}))}-1\right)\right\rangle+f(g(\bar{x}), y) \nless_{\operatorname{intC}(\bar{x})} 0, \forall y \in K .
\end{aligned}
$$

This completes the proof. 
Theorem 3.2. Let $\mathrm{K}$ be a nonempty closed convex and bounded subset of a real ordered Euclidean space $\mathrm{X}$. Let $\mathrm{C}: \mathrm{K} \rightarrow 2^{\mathrm{Y}}$ be a proper pointed closed convex cone valued mapping with $\operatorname{int} \mathrm{C} \neq \emptyset$ and $\mathrm{Y} \backslash(-\operatorname{int} \mathrm{C}(\mathrm{x}))$ being an upper semi continuous mapping. Let $\mathrm{g}: \mathrm{K} \rightarrow \mathrm{K}$ be a closed convex and continuous single valued mapping. Suppose $\eta: K \times K \rightarrow X$ is affine in the first argument with $\eta(x, g(x))=0$ for $x \in K$ and continuous in both variable. Let $\mathrm{f}: \mathrm{K} \times \mathrm{K} \rightarrow \mathrm{Y}$ be a completely continuous in the first argument and affine in the second argument satisfying $\mathrm{f}(\mathrm{g}(\mathrm{x}), \mathrm{x})=0$ for $\mathrm{x} \in \mathrm{K}$. Let $\alpha_{\mathrm{g}}: \mathrm{X} \rightarrow \mathrm{Y}$ be a weakly lower semicontinuous with respect to $\mathrm{g}$. Let $\mathrm{N}: \mathfrak{F}(\mathrm{L}(\mathrm{X}, \mathrm{Y})) \rightarrow \mathfrak{F}(\mathrm{L}(\mathrm{X}, \mathrm{Y}))$ be a continuous mapping and $\tilde{\mathrm{T}}: \mathrm{K} \rightarrow 2^{\mathrm{L}(\mathrm{X}, \mathrm{Y})}$ be a nonempty compact valued fuzzy mapping induced by fuzzy mapping $\mathrm{T}: \mathrm{K} \rightarrow \mathfrak{F}(\mathrm{L}(\mathrm{X}, \mathrm{Y}))$, i.e. $\tilde{\mathrm{T}}(\mathrm{x})=(\mathrm{T} x)_{\mathrm{a}(\mathrm{x})}$ and $\mathrm{a}: \mathrm{X} \rightarrow[0,1]$. If $\tilde{\mathrm{T}}$ is $\mathrm{H}$-hemicontinuous and $\alpha_{\mathrm{g}}$-relaxed exponentially $(\gamma, \eta)$-monotone with respect to $\mathrm{N}$ and $\mathrm{g}$, then $(2.1)$ is a solvable, i.e., there exist $\mathrm{x} \in \mathrm{K}$ and $\mathrm{u} \in \tilde{\mathrm{T}}(\mathrm{x})=(\mathrm{T} x)_{\mathrm{a}(\mathrm{x})}$ such that

$$
\left\langle\mathrm{Nu}, \frac{1}{\gamma}\left(e^{\gamma \eta(y, g(x))}-1\right)\right\rangle+f(g(x), y) \star_{\operatorname{int} C(x)} 0, \forall y \in K
$$

Proof. Let $\mathrm{F}: \mathrm{K} \rightarrow 2^{\mathrm{X}}$ be a set valued mapping define by

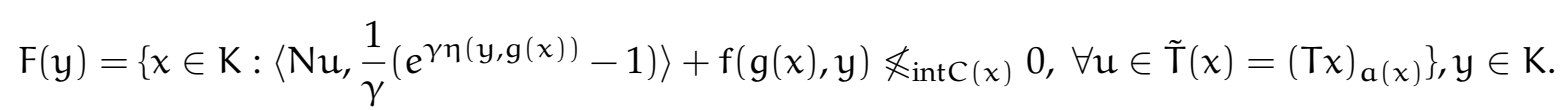

First we prove that $F$ is a KKM mapping. If $F$ is not a KKM-mapping, then there exists $\left(x_{1}, x_{2}, \ldots, x_{m}\right) \subset K$ such that

$$
\operatorname{co}\left\{x_{1}, x_{2}, \ldots, x_{m}\right\} \nsubseteq \bigcup_{i=1}^{m} F\left(x_{i}\right) .
$$

This implies that there exists at least an $x \in \operatorname{co}\left\{x_{1}, x_{2}, \ldots, x_{m}\right\}, x=\sum_{i=1}^{m} t_{i} x_{i}$, where $t_{i} \geqslant 0, i=1,2 \ldots, m$, $\sum_{i=1}^{m} t_{i}=1$, but $x \notin \bigcup_{i=1}^{m} F\left(x_{i}\right)$. From the construction of $F$, for any $u \in \tilde{T}(x)=(T x)_{a}(x)$, we have

$$
\left\langle N u, \frac{1}{\gamma}\left(e^{\gamma \eta\left(x_{i}, g(x)\right)}-1\right)\right\rangle+f\left(g(x), x_{i}\right) \leqslant \operatorname{intC}(x) 0, \text { for } i=1,2, \ldots, m .
$$

Since $\eta$ is affine in the first argument, it follows from (3.8) that

$$
\begin{aligned}
0= & \left\langle N u, \frac{1}{\gamma}\left(e^{\gamma \eta(x, g(x))}-1\right)\right\rangle+f(g(x), x) \\
& =\left\langle N u, \frac{1}{\gamma}\left(e^{\gamma \eta\left(\sum_{i=1}^{m} t_{i} x_{i}, g(x)\right)}-1\right)\right\rangle+f\left(g(x), \sum_{i=1}^{m} t_{i} x_{i}\right) \\
& =\left\langle N u, \frac{1}{\gamma}\left(e^{\sum_{i=1}^{m} t_{i} \gamma \eta\left(x_{i}, g(x)\right)}-1\right)\right\rangle+\sum_{i=1}^{m} t_{i} f\left(g(x), x_{i}\right) \\
& \leqslant C(x)\left\langle N u, \frac{1}{\gamma} \sum_{i=1}^{m} t_{i}\left(e^{\gamma \eta\left(x_{i}, g(x)\right)}-1\right)\right\rangle+\sum_{i=1}^{m} t_{i} f\left(g(x), x_{i}\right) \\
& =\sum_{i=1}^{m} t_{i}\left\{\left\langle N u, \frac{1}{\gamma}\left(e^{\gamma \eta\left(x_{i}, g(x)\right)}-1\right)\right\rangle+f\left(g(x), x_{i}\right)\right\} \leqslant \operatorname{intC}(x) 0,
\end{aligned}
$$

this shows that $0 \in \operatorname{int} C(x)$. This contradicts the fact that $C(x)$ is proper pointed closed convex cone valued mapping with int $C \neq \emptyset$. Hence $F$ is a KKM-mapping.

Now we define another set valued mapping $G: K \rightarrow 2^{\mathrm{X}}$ by

$$
G(y)=\left\{x \in K:\left\langle N v, \frac{1}{\gamma}\left(e^{\gamma \eta(y, g(x))}-1\right)\right\rangle+f(g(x), y) \mathbb{i n t C}_{(x)} \alpha_{g}(y-x), \quad \forall v \in \tilde{T}(y)\right\}, y \in K .
$$


We prove that $F(y) \subset G(y)$ for all $y \in K$. In fact, let $x \in F(y)$, there exists some $u \in \tilde{T}(x)=(T x)_{a(x)}$ such that

$$
\left\langle\mathrm{Nu}, \frac{1}{\gamma}\left(e^{\gamma \eta(y, g(x))}-1\right)\right\rangle+\mathrm{f}(\mathrm{g}(\mathrm{x}), \mathrm{y}) \mathbb{\mathrm { intC }}(\mathrm{x}) 0 .
$$

Since $\tilde{T}$ is a $\alpha_{g}$-relaxed exponentially $(\gamma, \eta)$-monotone with respect to $N$ and $g$, for any $y \in K, v \in \tilde{T}(y)=$ $(\mathrm{Ty})_{\mathrm{a}(\mathrm{y})}$, we have

$$
\left\langle N u, \frac{1}{\gamma}\left(e^{\gamma \eta(y, g(x))}-1\right)\right\rangle+f(g(x), y) \leqslant c(x)\left\langle N v, \frac{1}{\gamma}\left(e^{\gamma \eta(y, g(x))}-1\right)\right\rangle+f(g(x), y)-\alpha_{g}(y-x) .
$$

From (3.9), (3.10), and Lemma 2.1, we have

$$
\left\langle\mathrm{N} v, \frac{1}{\gamma}\left(e^{\gamma \eta(y, g(x))}-1\right)\right\rangle+f(g(x), y) \Varangle_{\operatorname{int} C(x)} \alpha_{g}(y-x), \quad \forall y \in K, v \in \tilde{T}(y)=(T y)_{a(y)} .
$$

Therefore $x \in G(y)$, i.e., $F(y) \subset G(y)$ for all $y \in K$. This implies that $G$ is also a KKM-mapping.

Next we prove that for each $y \in K, G(y) \subset K$ is closed in the weak topology of $X$.

In fact, if $\bar{x} \in \overline{G(y)}^{w}$, the weak closure of $G(y)$, since $X$ is reflexive, there is a sequence $\left\{x_{n}\right\}$ in $G(y)$ such that $\left\{x_{n}\right\}$ converges weakly to $\bar{x} \in K$. Therefore for each $v \in \tilde{T}(y)=(T y)_{a(y)}$, we have

$$
\left\langle N v, \frac{1}{\gamma}\left(e^{\gamma \eta\left(y, g\left(x_{n}\right)\right)}-1\right)\right\rangle+f\left(g\left(x_{n}\right), y\right) K_{i n t C}\left(x_{n}\right) \alpha_{g}\left(y-x_{n}\right),
$$

i.e.,

$$
\left\langle\mathrm{N} v, \frac{1}{\gamma}\left(e^{\gamma \eta\left(y, g\left(x_{n}\right)\right)}-1\right)\right\rangle+f\left(g\left(x_{n}\right), y\right)-\alpha_{g}\left(y-x_{n}\right) \in Y \backslash\left(-\operatorname{intC}\left(x_{n}\right)\right) .
$$

Since $N v, f$, and $g$ are completely continuous, $Y \backslash(-\operatorname{int} C(x))$ is closed and $\alpha_{g}$ is weakly lower semicontinuous, therefore the sequence

$$
\left\{\left\langle N v, \frac{1}{\gamma}\left(e^{\gamma \eta\left(y, g\left(x_{n}\right)\right)}-1\right)\right\rangle+f\left(g\left(x_{n}\right), y\right)-\alpha_{g}\left(y-x_{n}\right)\right\}
$$

converges to

$$
\left\langle N v, \frac{1}{\gamma}\left(e^{\gamma \eta(y, g(\bar{x}))}-1\right)\right\rangle+f(g(\bar{x}), y)-\alpha_{g}(y-\bar{x}),
$$

and

Therefore

$$
\left\langle\mathrm{N} v, \frac{1}{\gamma}\left(e^{\gamma \eta(y, g(\bar{x}))}-1\right)\right\rangle+f(g(\bar{x}), y)-\alpha_{g}(y-\bar{x}) \in Y \backslash(-\operatorname{int} C(\bar{x})) .
$$

$$
\left\langle N v, \frac{1}{\gamma}\left(e^{\gamma \eta(y, g(\bar{x}))}-1\right)\right\rangle+f(g(\bar{x}), y) \nless_{\operatorname{intC}(\bar{x})} \alpha_{g}(y-\bar{x})
$$

Hence $\bar{x} \in G(y)$. This proves that $G(y)$ is weakly closed for all $y \in K$.

Furthermore, since $X$ is reflexive, $K \subset X$ is a nonempty closed and convex bounded subset and $K$ is weakly compact, this implies that $\mathrm{G}(\mathrm{y})$ is also weakly compact. Therefore it follows from Lemma 2.6 and Theorem 3.1 that

$$
\bigcap_{y \in K} G(y) \neq \emptyset
$$

So there exists $\bar{x} \in K$ such that

$$
\left\langle\mathrm{N} v, \frac{1}{\gamma}\left(e^{\gamma \eta(y, g(\bar{x}))}-1\right)\right\rangle+\mathrm{f}(\mathrm{g}(\bar{x}), y) \mathbb{i n t C}_{(\bar{x})} \alpha_{g}(y-\bar{x}), \forall y \in K, v \in \tilde{T}(y)=(T y)_{a}(y) .
$$

Hence from Theorem 3.1, we can conclude that there exist $\bar{x} \in K$ and $\bar{u} \in \tilde{T}(\bar{x})=(T \bar{x})_{a(\bar{x})}$ such that

$$
\left\langle N \bar{u}, \frac{1}{\gamma}\left(e^{\gamma \eta(y, g(\bar{x}))}-1\right)\right\rangle+f(g(\bar{x}), y) \nless_{\operatorname{intC}(\bar{x})} 0, \forall y \in K,
$$

i.e., (2.1) is solvable. 
Theorem 3.3. Let $\mathrm{K}$ be a nonempty closed convex subset of a real ordered Euclidean space $\mathrm{X}$ with $0 \in \mathrm{K}$. Let $\mathrm{C}: \mathrm{K} \rightarrow 2^{\mathrm{Y}}$ be a proper pointed and closed convex cone valued mapping such that $\operatorname{int} \mathrm{C}(\mathrm{x}) \neq \emptyset$. Let $\mathrm{Y} \backslash(-\operatorname{int} \mathrm{C}(\mathrm{x}))$ be a upper semi continuous mapping and $\mathrm{g}: \mathrm{K} \rightarrow \mathrm{K}$ be a closed convex and continuous single valued mapping. Assume that $\eta: \mathrm{K} \times \mathrm{K} \rightarrow \mathrm{X}$ is affine in the first argument with $\eta(\mathrm{x}, \mathrm{g}(\mathrm{x}))=0$ for all $\mathrm{x} \in \mathrm{K}$. Let $\mathrm{f}: \mathrm{K} \times \mathrm{K} \rightarrow \mathrm{Y}$ be a completely continuous mapping in the first argument and affine in the second argument satisfying the condition $\mathrm{f}(\mathrm{x}, \mathrm{g}(\mathrm{x}))=0$ for all $\mathrm{x} \in \mathrm{K}$. Let $\alpha_{\mathrm{g}}: \mathrm{X} \rightarrow \mathrm{Y}$ be weakly lower semicontinuous. Let $\mathrm{N}: \mathfrak{F}(\mathrm{L}(\mathrm{X}, \mathrm{Y})) \rightarrow \mathfrak{F}\left(\mathrm{L}^{\mathrm{c}}(\mathrm{X}, \mathrm{Y})\right)$ be a continuous mapping, where $\mathrm{L}^{\mathrm{c}}(\mathrm{X}, \mathrm{Y})$ is a space of all completely continuous linear mapping from $\mathrm{X}$ to $\mathrm{Y}$ and $\tilde{\mathrm{T}}: \mathrm{K} \rightarrow 2^{\mathrm{L}(\mathrm{X}, \mathrm{Y})}$ is a nonempty compact valued and upper semi continuous mapping induced by a fuzzy mapping $\mathrm{T}: \mathrm{K} \rightarrow \mathfrak{F}(\mathrm{L}(\mathrm{X}, \mathrm{Y}))$, i.e., $\tilde{\mathrm{T}}(\mathrm{x})=(\mathrm{T} x)_{\mathrm{a}(\mathrm{x})}$ for $\mathrm{x} \in \mathrm{K}$. If $\tilde{\mathrm{T}}$ is $\mathrm{H}$-hemicontinuous and $\alpha_{\mathrm{g}}$-relaxed exponentially $(\gamma, \eta)$-monotone with respect to $\mathrm{N}$ and $\mathrm{g}$ and there exists $\mathrm{r}>0$ such that

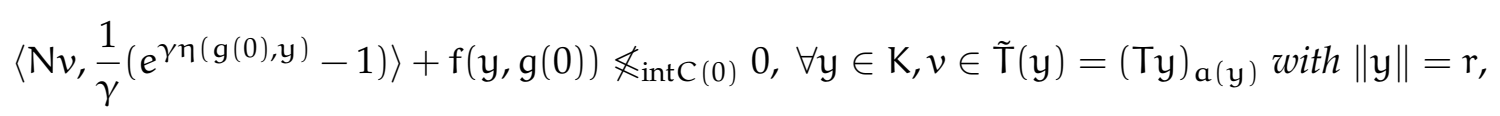

then (2.1) is a solvable, i.e., there exists $\mathrm{x} \in \mathrm{K}$ and $\mathrm{u} \in \tilde{\mathrm{T}}(\mathrm{x})=(\mathrm{T} x)_{\mathbf{a}(\mathrm{x})}$ such that

$$
\left\langle\mathrm{Nu}, \frac{1}{\gamma}\left(e^{\gamma \eta(y, g(x))}-1\right)\right\rangle+\mathrm{f}(\mathrm{g}(\mathrm{x}), \mathrm{y}) \mathbb{\mathrm { intC }}_{\mathrm{in}(\mathrm{x})} 0, \forall \mathrm{y} \in \mathrm{K}
$$

Proof. For $r>0$, denote by $K_{r}=\{x \in X,\|x\| \leqslant r\}$. From Theorem 3.2, we know that (2.1) is solvable over $K_{r}$, i.e., there exists $x_{r} \in K \cap K_{r}$ and $u_{r} \in \tilde{T}\left(x_{r}\right)=\left(T x_{r}\right)_{a\left(x_{r}\right)}$ such that

$$
\left\langle N u_{r}, \frac{1}{\gamma}\left(e^{\gamma \eta\left(y, g\left(x_{r}\right)\right)}-1\right)\right\rangle+f\left(g\left(x_{r}\right), y\right) z_{\operatorname{intC}\left(x_{r}\right)} 0, \forall y \in K \bigcap K_{r} .
$$

Putting $y=0$ in (3.12) we have

$$
\left\langle N u_{r}, \frac{1}{\gamma}\left(e^{\gamma \eta\left(0, g\left(x_{r}\right)\right)}-1\right)\right\rangle+f\left(g\left(x_{r}\right), 0\right) \leqslant \operatorname{intC}\left(x_{r}\right) 0 .
$$

If $\left\|x_{r}\right\|=r$, then it contradicts to (3.11). Hence $r>\left\|x_{r}\right\|$. For any $z \in K$, let us choose $t \in(0,1)$ small enough such that $(1-t) x_{r}+t z \in K \cap K_{r}$. Putting $y=(1-t) x_{r}+t z$ in (3.12), we get

$$
\left\langle N u_{r}, \frac{1}{\gamma}\left(e^{\gamma \eta\left((1-t) x_{r}+t z, g\left(x_{r}\right)\right)}-1\right)\right\rangle+f\left(g\left(x_{r}\right),(1-t) x_{r}+t z\right) \Varangle_{i n t C}\left(x_{r}\right) 0 .
$$

Since $\eta$ is affine in the first variable, we have

$$
\begin{aligned}
\left\langle N u_{r},\right. & \left.\frac{1}{\gamma}\left(e^{\gamma \eta\left((1-t) x_{r}+t z, g\left(x_{r}\right)\right)}-1\right)\right\rangle+f\left(g\left(x_{r}\right),(1-t) x_{r}+t z\right) \\
& =\left\langle N u_{r}, \frac{1}{\gamma}\left(e^{(1-t) \gamma \eta\left(x_{r}, g\left(x_{r}\right)\right)+t \gamma \eta\left(z, g\left(x_{r}\right)\right)}-1\right)\right\rangle+\mathrm{tf}\left(g\left(x_{r}\right), z\right) \\
& \leqslant \mathrm{C}\left(x_{r}\right)\left\langle N u_{r}, \frac{1}{\gamma}(1-t)\left(e^{\gamma \eta\left(x_{r}, g\left(x_{r}\right)\right)}-1\right)+\frac{1}{\gamma} \mathrm{t}\left(e^{\gamma \eta\left(z, g\left(x_{r}\right)\right)}-1\right)\right\rangle+\mathrm{tf}\left(g\left(x_{r}\right), z\right) \\
& =\mathrm{t}\left\{\left\langle N u_{r}, \frac{1}{\gamma}\left(e^{\gamma \eta\left(z, g\left(x_{r}\right)\right)}-1\right)\right\rangle+\mathrm{tf}\left(g\left(x_{r}\right), z\right)\right\} .
\end{aligned}
$$

Hence from (3.13), (3.14), and Lemma 2.1, we get

$$
\left\langle\mathrm{Nu} u_{r}, \frac{1}{\gamma}\left(e^{\gamma \eta\left(z, g\left(x_{r}\right)\right)}-1\right)\right\rangle+f\left(g\left(x_{r}\right), z\right) \Varangle_{\operatorname{intC}\left(x_{r}\right)} 0, \forall z \in K .
$$

Therefore, (2.1) is a solvable. This completes the proof. 


\section{Acknowledgment}

The first author was supported by the Natural Science Foundation of China Medical University, Taiwan and the Research Center for Nonlinear Analysis and Optimization, Kaohsiung Medical University, Taiwan. The third author was supported by the Research Center for Nonlinear Analysis and Optimization, Kaohsiung Medical University, Taiwan and partially supported by Grant MOST 106-2115-M-037-001.

\section{References}

[1] M. K. Ahmad, S. Salahuddin, A fuzzy extension of generalized implicit vector variational like inequalities, Positivity, 11 (2007), 477-484. 1

[2] M. K. Ahmad, S. Salahuddin, Existence of solution for generalized implicit vector variational like inequalities, Nonlinear Anal., 67 (2007), 430-441. 1

[3] M. K. Ahmad, S. Salahuddin, Fuzzy generalized variational like inequality problems in topological vector spaces, J. Fuzzy Set Valued Anal., 2013 (2013), 5 pages. 1

[4] G. A. Anastassiou, S. Salahuddin, Weakly set valued generalized vector variational inequalities, J. Comput. Anal. Appl., 15 (2013), 622-632. 1

[5] L. Q. Anh, P. Q. Khanh, Existence conditions in symmetric multivalued vector quasiequilibrium problems, Control Cybernet., 36 (2007), 519-530. 1

[6] L. E. J. Brouwer, Zur invarianz des n-dimensional gebietes, Math. Ann., 71 (1912), 305-313. 1, 2.12, 2.14, 2.15

[7] L. C. Ceng, J. C. Yao, On generalized variational-like inequalities with generalized monotone multivalued mappings, Appl. Math. Lett., 22 (2009), 428-434. 1, 2.1

[8] C. L. Chang, Fuzzy topological spaces, J. Math. Anal. Appl., 24 (1968), 182-190. 1

[9] S. S. Chang, S. Salahuddin, Existence of vector quasi variational like inequalities for fuzzy mappings, Fuzzy Sets and Systems, 233 (2013), 89-95. 1

[10] S. S. Chang, S. Salahuddin, M. K. Ahmad, X. R. Wang, Generalized vector variational like inequalities in fuzzy environment, Fuzzy Sets and System, 265 (2015), 110-120. 1, 2.15

[11] S.-S. Chang, Y.-G. Zhu, On variational inequalities for fuzzy mappings, Fuzzy Sets and Systems, 32 (1989), 359-367. 1

[12] G. Y. Chen, Existence of solutions for a vector variational inequalities: An extension of Hartman-Stampacchia theorems, J. Optim. Theory Appl., 74 (1992), 445-456. 1

[13] G.-Y. Chen, G.-M. Cheng, Vector variational inequality and vector optimizations problem, Toward Interactive and Intelligent Decision Support Systems, Lecture Notes in Economics and Mathematical Systems, Springer, Berlin, Heidelberg, (1987). 1

[14] Y. J. Cho, S. Salahuddin, M. K. Ahmad, A fuzzy extension of generalized vector version of Minty lemma and applications, J. Fuzzy Maths., 15 (2007), 449-458. 1

[15] X. P. Ding, S. Salahuddin, Fuzzy generalized mixed vector quasi variational like inequalities, J. Emerging Trends Comput. Information Sci., 4 (2013), 881-887. 1

[16] X. P. Ding, S. Salahuddin, M. K. Ahmad, Fuzzy generalized vector variational inequalities and complementarity problems, Nonlinear Funct. Anal. Appl., 13 (2008), 253-263. 1

[17] K. Fan, A generalization of tychonoff's fixed point theorem, Math. Ann., 142 (1961), 305-310. 2.6

[18] Y. P. Fang, N. J. Huang, Variational-like inequalities with generalized monotone mappings in Banach spaces, J. Optim. Theory Appl., 118 (2003), 327-338. 1

[19] F. Giannessi, Theorems of the Alternative, Quadratic Programs, and Complementarity Problems, Variational Inequalities and Complementarity Problems, Edited by R. W. Cottle, F. Giannessi, and J. L. Lions, John Wiley and Sons, New York, (1980). 1

[20] N.-J. Huang, C.-J. Gao, Some generalized vector variational inequalities and complementarity problems for multivalued mappings, Appl. Math. Lett., 16 (2003), 1003-1010. 1

[21] A. Jayswal, S. Choudhury, R. U. Verma, Exponential type vector variational-like inequalities and vector optimization problems with exponential type invexities, J. Appl. Math. Comput., 45 (2014), 87-97. 1

[22] P. Q. Khanh, N. H. Quan, Generic stability and essential components of generalized KKM points and applications, J. Optim. Theory Appl., 148 (2011), 488-504. 1

[23] B. S. Lee, S. Salahuddin, A hemivariational-like inequality with applications, J. Nonlinear Convex Anal., 16 (2015), 141-150. 1

[24] B. S. Lee, S. Salahuddin, Minty lemma for inverted vector variational inequalities, Optimization, 66 (2017), 351-359. 1

[25] K. L. Lin, D.-P. Yang, J. C. Yao, Generalized vector variational inequalities, J. Optim. Theory Appl., 92 (1997), 117-125. 1

[26] S. B. J. Nadler, Multi-valued contraction mappings, Pacific J. Math., 30 (1969), 475-488. 1, 2.7

[27] S. Salahuddin, M. K. Ahmad, R. U. Verma, Existence theorem for fuzzy mixed vector F-variational inequalities, Adv. Nonlinear Var. Inequal., 16 (2013), 53-59. 1

[28] S. Salahuddin, B. S. Lee, Fuzzy general nonlinear ordered random variational inequalities in ordered Banach spaces, East Asian Math. J., 32 (2016), 685-700. 1 
[29] S. Salahuddin, R. U. Verma, A common fixed point theorem for fuzzy mappings, Trans. Math. Prog. Appls., 1 (2013), 59-68. 1

[30] A. H. Siddiqi, M. F. Khan, S. Salahuddin, On vector variational like inequalities, Far East J. Math. Sci., Special Volume, Part III, (1998), 319-329. 1

[31] K.-Q. Wu, N.-J. Huang, Vector variational-like inequalities with relaxed $\eta-\alpha$-Pseudomonotone mappings in Banach spaces, J. Math. Inequal., 1 (2007), 281-290. 1

[32] L. A. Zadeh, Fuzzy sets, Information Control, 8 (1964), 338-353. 1 